\title{
Diplejía facial como manifestación de neuroleptospirosis
}

\section{Facial diplegia as a manifestation of neuroleptospirosis}

\author{
Jenny Patricia Muñoz-Lombo, Diana Carolina Quintero-González, \\ José Mauricio Cárdenas-Prieto, María Eugenia Casanova-Valderrama • Cali (Colombia)
}

DOI: https://doi.org/10.36104/amc.2021.1947

\section{Resumen}

Esta serie de caso describe los hallazgos clínicos y paraclínicos observados en dos hombres jóvenes, con parálisis facial periférica bilateral o diplejía facial durante la fase de convalecencia de la leptospirosis, con recuperación neurológica sin secuela; resaltando así, el papel de la espiroqueta en el desarrollo de una variante atípica y poco frecuente del síndrome de Guillain-Barré. (Acta Med Colomb 2021; 46. DOI: https://doi.org/10.36104/amc.2021.1947).

Palabras clave: síndrome febril, leptospirosis, manifestaciones neurológicas, diplejía facial, síndrome de Guillain-Barré, Colombia.

\begin{abstract}
This case series describes the clinical and paraclinical findings in two young men with bilateral peripheral facial palsy or facial diplegia during the convalescent period of leptospirosis, who recovered neurologically without sequelae. This highlights the role of spirochetes in the development of an atypical and rare variant of Guillain-Barré syndrome. (Acta Med Colomb 2021; 46. DOI: https://doi.org/10.36104/amc.2021.1947).
\end{abstract}

Key words: febrile syndrome, leptospirosis, neurological manifestations, facial diplegia, Guillain-Barre syndrome, Colombia.
Dr. Jenny Patricia Muñoz-Lombo: Internista, Magister en Epidemiología, Universidad Libre seccional Cali, Clínica Nuestra Señora de los Remedios; Dra. Diana Carolina Quintero-González: Internista, Magister en Epidemiología, Universidad Libre Seccional Cali, Clínica Nuestra Señora de los Remedios; Dr. José Mauricio Cárdenas-Prieto: Internista, Universidad Libre Seccional Cali, Gesencro Buga; Dra. María Eugenia Casanova-Valderrama: Internista y Neurólogo Clínico. Directora del programa de Medicina Interna Universidad Libre Cali, Grupo Interinstitucional de Medicina Interna (GIMI 1), Dime Clínica Neurocardiovascular. Cali (Colombia).

Correspondencia: Dr. Jenny Patricia MuñozLombo. Cali (Colombia).

E-mail: ideasenproceso@hotmail.com

Recibido: 13/VII/2020 Aceptado: 22/XII/2020

\section{Introducción}

La parálisis facial periférica bilateral o diplejía facial (DF) es una rara manifestación neurológica donde cada nervio facial se afecta de manera simultánea o con un intervalo menor a 30 días, representando menos del $2 \%$ de los casos de parálisis facial, con una incidencia mundial estimada de un caso por cada cinco millones de habitantes. La causa más común es el síndrome de Guillain-Barré (SGB), una polineuroradiculopatía de inicio agudo (1), donde la paresia facial se desarrolla entre 24 y $60 \%$, en la mayoría de los casos bilateral. La DF aislada sin compromiso o escaso de extremidades, es una variante atípica y poco frecuente del SGB $(2,3)$. Varios tipos de infecciones han sido asociadas al SGB, principalmente de tipo respiratorio y gastrointestinal en $70 \%$ de los casos. La asociación con leptospirosis una de las zoonosis de mayor distribución mundial generada por la espiroqueta del orden Spirochaetales, de la familia Leptospiraceae, del género Leptospira se ha documentado con menor frecuencia (4-7). Esta infección en la mayoría de los casos tiene una presentación clínica bifásica que co- mienza con un fase septicémica, seguida de manifestaciones inmunes con un espectro clínico amplio que va desde un síndrome febril con síntomas constitucionales leves hasta un cuadro severo acompañado de falla multiorgánica (8). Tiene tropismo por algunos órganos como el hígado, riñones, corazón y músculo esquelético, pero además manifestaciones neurológicas entre $12-40 \%$, tales como meningoencefalitis aséptica, accidente cerebrovascular, mono o polineuritis, parálisis de los nervios craneales, mielopatía inflamatoria, radiculopatía y el ya mencionado $\operatorname{SGB}(9,10)$. El objetivo de este artículo es describir el comportamiento de dos casos inusuales de pacientes con leptospirosis que desarrollaron en el curso de la enfermedad, parálisis facial bilateral como variante atípica del SGB, debido al reto diagnóstico que esta patología representa para el clínico.

\section{Caso 1}

\section{Casos clínicos}

Hombre de 27 años, sin antecedentes relevantes, consultó a urgencias por cuadro clínico de nueve días de alzas 
térmicas no cuantificadas, escalofríos, cefalea, dolor abdominal y mialgia que posteriormente presentó parálisis facial periférica derecha y al día siguiente compromiso contralateral asociado a parestesias en extremidades superiores. Al examen físico, signos vitales normales, sin alteración de las funciones mentales superiores, con biparesia facial periférica sin compromiso de otros nervios craneales, fuerza global en extremidades $5 / 5$, reflejos miotendinosos normales y respuesta plantar flexora bilateral, la tomografía de cráneo simple de ingreso fue reportada dentro de límites normales, hemograma con leucocitosis (12 800 células $\left./ \mathrm{mm}^{3}\right) \sin$ neutrofilia, función renal y hepática normal, serología para Leptospira IgM positiva e IgG negativa, con lo cual se dio el diagnóstico de leptospirosis iniciando tratamiento con ceftriaxona 1 gramo endovenoso cada 12 horas por siete días. Debido a la persistencia de los hallazgos neurológicos se realizó una resonancia magnética cerebral simple y contrastada que fue normal, mientras que el líquido cefalorraquídeo (LCR) demostró una disociación albumino-citológica (Tabla 1, Caso 1) sugiriendo al SGB en su variante atípica diplejía facial, realizando electromiografía más velocidad de conducción de cada nervio facial que apoya el diagnóstico al evidenciar una neuropatía desmielinizante bilateral con signos de reinervación. El paciente se sometió a terapia de rehabilitación física y seguimiento por fonoaudiología presentando recuperación paulatina sin compromiso de la función deglutoria, respiratoria o debilidad en extremidades.

\section{Caso 2}

Hombre de 36 años, quien se desempeñaba como conductor de autobús internacional, sin antecedentes relevantes, consultó a urgencias por cuadro clínico de cinco días de fiebre de $38.8^{\circ} \mathrm{C}$, escalofríos, mialgias, artralgias,

Tabla 1. Punción lumbar.

\begin{tabular}{|c|c|c|}
\hline Casos & 1 & 2 \\
\hline \multicolumn{3}{|c|}{ Citoquímico de LCR } \\
\hline Color del líquido & Agua de roca & Xantocrómico \\
\hline pH & 6.0 & 8.0 \\
\hline Densidad & 1.010 & 1.010 \\
\hline \multicolumn{3}{|l|}{ Examen citológico } \\
\hline Leucocitos & $5 \mathrm{cel} / \mathrm{mm}^{3}$ & $8 \mathrm{cel} / \mathrm{mm}^{3}$ \\
\hline Linfocitos & $5(100 \%)$ & 5 \\
\hline Eritrocitos & 0 & $\begin{array}{l}51 / \mathrm{mm}^{3} \text { hematíes frescos } \\
100 \%\end{array}$ \\
\hline \multicolumn{3}{|c|}{ Examen químico } \\
\hline Glucosa & $59 \mathrm{mg} / \mathrm{dL}$ & $59 \mathrm{mg} / \mathrm{dL}$ \\
\hline Proteínas & $135 \mathrm{mg} / \mathrm{dL}$ & $223 \mathrm{mg} / \mathrm{dL}$ \\
\hline LDH & $200 \mathrm{U} / \mathrm{dl}(0-400 \mathrm{U} / \mathrm{dl})$ & $111 \mathrm{U} / \mathrm{dl}(0-400 \mathrm{U} / \mathrm{dl})$ \\
\hline Perfil infeccioso & $\begin{array}{l}\text { Gram. BK. KOH. tinta } \\
\text { china. VDRL. cultivo de } \\
\text { LCR à Negativos }\end{array}$ & $\begin{array}{l}\text { Gram. BK. KOH. tinta } \\
\text { china. VDRL. cultivo de } \\
\text { LCR à Negativos }\end{array}$ \\
\hline Glucosa sérica & $82 \mathrm{mg} / \mathrm{dL}$ & $94 \mathrm{mg} / \mathrm{dL}$ \\
\hline
\end{tabular}

dolor abdominal generalizado tipo cólico de intensidad variable y epistaxis autolimitada. Al examen físico ictericia mucocutánea generalizada, deshidratación leve, dolor a la palpación en epigastrio e hipocondrio derecho con hepatoesplenomegalia confirmada con ecografía abdominal total. La bioquímica sanguínea mostró hemoconcentración (relación hemoglobina/hematocrito $=42.7 \% / 12.6 \mathrm{~g} / \mathrm{dL}=3.38$ ), trombocitopenia $\left(73000 / \mathrm{mm}^{3}\right)$, hiperbilirrubinemia a expensas de la directa (totales $5.2 \mathrm{mg} / \mathrm{dL}$, directa $3.37 \mathrm{mg} / \mathrm{dL}$ ), elevación de transaminasas (ALT $165 \mathrm{U} / \mathrm{L}, \mathrm{AST} 150 \mathrm{U} / \mathrm{L} \mathrm{mg} /$ dL), fosfatasa alcalina (237 IU/I) y deshidrogenasa láctica (1098 IU/I) además de hematuria eumórfica microscópica (10-15xc) con proteinuria (2+), con control en 24 horas con leucocitosis con neutrofilia de (WBC 12200 células $/ \mathrm{mm}^{3}$, neutrófilos $80 \%$ ) descenso plaquetario $\left(35000 \mathrm{~mm}^{3}\right)$, PCR elevada $(44 \mathrm{mg} / \mathrm{dL})$ y lesión renal aguda AKIN I (Cr 1.7 $\mathrm{mg} / \mathrm{dL}$ ), se solicitó perfil infeccioso el cual fue negativo (serología para Leptospira IgM e IgG, dengue, hepatitis A, B y C, VIH, sífilis, malaria y bacteriemia); sin embargo, dada la clínica y los paraclínicos se dio impresión diagnóstica de síndrome de Weil iniciando manejo empírico con ceftriaxona 1 gramo endovenoso cada 12 horas más terapia de reanimación hídrica solicitando prueba de aglutinación microscópica para leptospira (MAT), que confirmó el diagnóstico al presentar un resultado positivo para el serogrupo australis 1:200 y serogrupo cynopteri 1:400. Al décimo día de inicio de los síntomas presenta parálisis facial izquierda periférica con compromiso contralateral a las 24 horas (diplejía facial), sin otros hallazgos relevantes al examen físico; la tomografía de cráneo simple fue normal mientras que el líquido cefalorraquídeo (LCR) demostró una disociación albumino-citológica (Tabla 1, Caso 2), sugiriendo un SGB variante DF clasificación Barré Hughes 1 sin progresión del déficit neurológico, por lo que no se inició tratamiento con inmunoglobulina o plasmaféresis. El paciente fue egresado con terapia de rehabilitación y glucocorticoides con controles por neurología clínica. La DF se resolvió gradualmente después de la tercera semana de tratamiento.

\section{Discusión}

Presentamos el caso de dos pacientes con leptospirosis en escenarios clínicos diferentes, el primero en una leptospirosis clásica y el segundo en contexto de un síndrome de Weil, quienes durante la fase de convalecencia presentaron biparesia facial periférica sin compromiso de extremidades, como complicación neurológica poco frecuente, en contexto de variante atípica del SGB. Esta polineuroradiculopatía inmuno-mediada, es considerada la causa más frecuente de parálisis flácida aguda en el mundo luego de la erradicación de la poliomielitis (11), tiene en sus formas más comunes a la AIDP (del inglés, Acute inflammatory demyelinating polyneuropathy), la AMAN (del inglés, acute motor axonal neuropathy) y la AMSAN (del inglés, acute motor and sensory axonal neuropathy). Sin embargo, existen variantes de presentación atípicas como el Síndrome de Miller Fisher 
(SMF), la encefalitis de Bickerstaff, la variante faríngeacervical-braquial (FCB), la parálisis del sexto par y la DF que dificultan el reconocimiento de la enfermedad y suponen un reto diagnóstico (12). Un estudio prospectivo de 250 pacientes con SGB encontró, que el SMF (5\%) y la variante FCB (3\%) son los subtipos más frecuentes mientras que la DF aislada se ha descrito en el $1 \%$ de los pacientes (13).

Se ha identificado la asociación de diferentes agentes infecciosos virales (Cytomegalovirus, Epstein-Barr, influenza A, Enterovirus, Zika) y bacterianos (Mycoplasma pneumoniae, Haemophilus influenzae, Campylo- bacter jejuni) en la inducción de la respuesta inmunológica tanto humoral como celular para la creación de anticuerpos de tipo IgG1 e IgG3 contra los axones y la mielina, debido al mimetismo molecular entre los oligosacáridos de los organismos infecciosos y los gangliosidos de la membrana neuronal (GM1-GD1a) (14). Sin embargo, para el caso de la leptospirosis la fisiopatología del compromiso neurológico es aún desconocida. Existen hipótesis que plantean la presencia de mecanismos inmunológicos como una explicación al polimorfismo de manifestaciones clínicas dadas por esta enfermedad, entre ellas la presencia de vasculitis sistémicas con activación de complejos inmunes circulantes que se desarrollan los días $6-15$ de iniciados los síntomas $(15,16)$. A su vez la reacción cruzada contra la vaina de mielina de los nervios periféricos (17) zoonosis bacteriana de distribución mundial. La leptospirosis humana resulta del contacto con agua, suelo o alimentos contaminados con la orina de ratas, perros, cerdos y otros animales leptospirúricos. Las leptospiras móviles penetran por la piel rota o a través de las superficies mucosas, y generan una enfermedad aguda y sistémica que se ha caracterizado por fiebre de inicio brusco, mialgias, cefalea intensa y hemorragias conjuntivales. Las manifestaciones clínicas de la enfermedad no son patognomónicas, por tanto, la leptospirosis ha sido diagnosticada erróneamente con mucha frecuencia. La mayoría de los casos humanos son leves y anictéricos; sin embargo, de 5 a $30 \%$ de los casos ictéricos pueden ser mortales, debido a complicaciones hemorrágicas, meningitis aséptica e insuficiencia renal (síndrome de Weil, la degeneración Walleriana e infiltraciones inflamatorias perivasculares y perineurales descrita en biopsias neuromusculares de pacientes con leptospirosis validan la asociación del SGB como complicación de la infección por esta espiroqueta $(17,18)$.

Se ha identificado una asociada con el SGB en una búsqueda de la literatura de los últimos 20 años, en la cual solo se encontraron ocho reportes de casos de parálisis facial asociada incluidas tres formas bilaterales de la enfermedad, en donde el compromiso neurológico y tiempo de evolución son comparables como los casos notificados en este reporte (19-22). A la fecha no se dispone de literatura sobre casos de leptospirosis asociados a la variante atípica DF del SGB, posiblemente a la falta de estudios en búsqueda de esta condición.

Para realizar el diagnóstico del SGB se puede basar en los criterios modificados de Wakerley et al, donde ciertas características clínicas, incluyendo la historia o antecedente de síntomas infecciosos, presencia de debilidad facial bilateral, parestesias distales, disminución o ausencia de reflejos tendinosos profundos y el curso monofónico de la enfermedad con un intervalo entre el inicio del déficit neurológico y el nadir de este, entre 12 horas y cuatro semanas son fuertemente sugestivos de SGB variante DF (23).

En la actualidad, no hay marcadores séricos, en orina o LCR que confirmen la enfermedad; sin embargo, la disociación albúmina-citológica (conteo de células normales con elevación de proteínas) evidente en el LCR es un hallazgo que orienta hacia el diagnóstico del SGB (24). En un estudio donde se incluyeron muestras de LCR de 474 pacientes con SGB se encontró que la sensibilidad de la disociación albumino-citológica depende del tiempo en que se toma la muestra, siendo positiva en $49 \%$ en el primer día de la enfermedad y puede aumentar a $88 \%$ después de la segunda semana (25). La pleoicitosis (leucocitos mayores a cinco en LCR) es inusual en el SGB, pero aproximadamente 15\% de los pacientes pueden tener conteo de leucocitos entre $5 \mathrm{y}$ 50 células por campo de alto poder como se evidenció en el según caso presentado (26).

\section{Conclusión}

La leptospirosis es una zoonosis reemergente en el mundo y la segunda causa de síndrome febril en Colombia. Si bien el compromiso renal, hepático y pulmonar son más frecuentes, las complicaciones neurológicas pueden manifestarse. Por lo tanto, si se presenta diplejía facial, el clínico debería realizar estudios del líquido cefalorraquídeo para la búsqueda de disociación albumino-citológica que permitan el diagnóstico del SGB en esta variante atípica y poco frecuente de la enfermedad.

\section{Referencias}

1. JR K. Bilateral seventh nerve palsy: analysis of 43 cases and review of the literature. Neurology. 1994;44(7):1198-202.

2. Varol S, Ozdemir HH, Akil E, Arslan D, Aluclu MU, Demir CF, et al. Facial diplegia: etiology, clinical manifestations, and diagnostic evaluation. Arq Neuropsiquiatr. 2015;73(12):998-1001.

3. Lehmann HC, MacHt S, Jander S, Hartung HP, Methner A. Guillain-Barré syndrome variant with prominent facial diplegia, limb paresthesia, and brisk reflexes. J Neurol. 2012;259(2):370-1.

4. Torres-Castro M, Hernández-Betancourt S, Agudelo-Flórez P, ArroyaveSierra E, Zavala-Castro J, Puerto FI. Articulos de revisión Revisión actual de la epidemiología de la leptospirosis Current review of the epidemiology of leptospirosis. Vol. 54, Rev Med Inst Mex Seguro Soc. 2016.

5. Adler B, de la Peña Moctezuma A. Leptospira and leptospirosis. Vet Microbiol. 2010;140(3-4):287-96.

6. Kobawaka Gamage KK, Fernando H. Leptospirosis complicated with Guillain Barre syndrome, papillitis and thrombotic thrombocytopenic Purpura; A case report. BMC Infect Dis. 2018;18(1):1-4.

7. Dev N, Kumar R, Kumar D. Guillain-Barre syndrome: a rare complication of leptospirosis and scrub typhus co-infection. Trop Doct. 2019;3-4.

8. El Bouazzaoui A, Houari N, Arika A, Belhoucine I, Boukatta B, Sbai H, et al. Facial palsy associated with leptospirosis. Eur Ann Otorhinolaryngol Head Neck Dis. 2011 Nov;128(5):275-7.

9. Levett PN. Leptospirosis: A forgotten zoonosis? Vol. 4, Clinical and Applied Immunology Reviews. Elsevier; 2004. p. 435-48.

10. Gaillard T, Martinaud C, Faivre A, Souraud JB, Maslin J, Alla P, et al. Moyens 
biologiques et stratégie diagnostique de la leptospirose neuroméningée. À propos d'un cas. Rev Med Interne. 2009 Apr;30(4):361-4.

11. Lehmann HC, Macht S, Jander S, Hartung HP MA. Guillain-Barré syndrome variant with prominent facial diplegia, limb paresthesia, and brisk reflexes. $J$ Neurol. 2012;259(2):370-1

12. Mazen M. Dimachkie, M.D., Richard J. Barohn M, Dimachkie, Mazen M. et al. Guillain-Barré Syndrome and Variants Mazen. Neurol Clin. 2014;31(2):491-510.

13. When is facial diplegia regarded as a variant of Guillain-Barré syndrome? $J$ Peripher Nerv Syst. 2015;20(1):32-6.

14. Eelco F.M. Wijdicks CJK. Guillain-Barré Syndrome. Mayo Clin Proc. 2017;92(3):467-79.

15. Thukral A, Khan I, Tripathi K. Slurred speech and spirochaetes. Lancet. 2009;373(9667):978.

16. de Souza AL, Sztajnbok J, Spichler A, Carvalho SM, de Oliveira ACP, Seguro AC. Peripheral nerve palsy in a case of leptospirosis. Trans R Soc Trop Med Hyg. $2006 \mathrm{Jul} ; 100(7): 701-3$

17. Carrada T. Leptospirosis humana. Historia natural, diagnóstico y tratamiento Leptospirosis. Rev Mex Patol Clin. 2005;52(4):246-56.

18. P.Azouvi, T. Hostachy, M. Desi et al. Polyneuropathie axonale aigue et réversible dans les suites d'une leptospirose. Rev Neurol. 1989;145:805-7.

19. Maldonado F, Portier H, Kisterman JP. Bilateral facial palsy in a case of leptospirosis. Scand J Infect Dis. 2004;36(5):386-8.

20. Andosilla LM, Brun MF, Miranda Col CC, Alberto Lora Andosilla M, Macia
Brun F, Arturo Cassiani Miranda C, et al. Parálisis facial periférica bilateral como presentación inicial del síndrome de Guillain-Barré: informe de un caso Bilateral facial nerve paralysis as initial manifestation of guillain-barré syndrome: a case report Caso clínico ActA NeurológicA colombiANA [Internet]. Vol. 31, Acta Neurol Colomb. 2015 [cited 2019 Aug 4]. Available from: http://www.scielo. org.co/pdf/anco/v31n4/v31n4a13.pdf

21. Costa E, Sacramento E, Lopes AA, Bina JC. Facial nerve palsy associated with leptospirosis. Rev Soc Bras Med Trop [Internet]. 2001 Apr [cited 2019 Sep 9];34(2):219-20. Available from: http://www.scielo.br/scielo.php?script=sci_ arttext\&pid=S0037-86822001000200011\&lng=en\&tlng=en

22. Midon A, Corrêa FB, Maia RD, Sampaio AG, Rosa Júnior M. Bilateral facial paralysis associated with leptospirosis. Vol. 51, Radiologia Brasileira. Colegio Brasileiro de Radiologia; 2018. p. 335.

23. Wakerley BR, Uncini A, Yuki N. Guillain-Barré and miller fisher syndromes New diagnostic classification. Nat Rev Neurol. 2014;10(9):537-44.

24. Wong AHY, Umapathi T, Nishimoto Y, Wang YZ, Chan YC, Yuki N. Cytoalbuminologic dissociation in Asian patients with Guillain-Barré and Miller Fisher syndromes. J Peripher Nerv Syst. 2015;20(1):47-51.

25. Fokke C, Van Den Berg B, Drenthen J, Walgaard C, Van Doorn PA, Jacobs BC. Diagnosis of Guillain-Barré syndrome and validation of Brighton criteria. Brain. 2014;137(1):33-43.

26. Esposito S, Longo MR. Guillain-Barré syndrome. Autoimmun Rev. 2017;16(1):96-101. 